\title{
Overcoming Obstacles to Drug Repositioning in Japan
}

\author{
Yuhei Nishimura ${ }^{1 *}$, Masaaki Tagawa ${ }^{2}$, Hideki Ito ${ }^{3}$, Kazuhiro Tsuruma $^{4}$ and Hideaki Hara ${ }^{4}$ \\ ${ }^{1}$ Department of Integrative Pharmacology, Mie University Graduate School of Medicine, Tsu, Japan, ${ }^{2}$ Medical Affairs, \\ Sumitomo Dainippon Pharma Co., Ltd., Tokyo, Japan, ${ }^{3}$ Department of CNS Research, Otsuka Pharmaceutical Co., Ltd., \\ Tokushima, Japan, ${ }^{4}$ Molecular Pharmacology, Department of Biofunctional Evaluation, Gifu Pharmaceutical University, Gifu, \\ Japan
}

Drug repositioning (DR) is the process of identifying new indications for existing drugs. DR usually focuses on drugs that have cleared phase-I safety trials but has yet to show efficacy for the intended indication. Therefore, DR can probably skip the preclinical and phase-I study, which can reduce the cost throughout drug development. However, the expensive phase-II/III trials are required to establish efficacy. The obstacles to DR include identification of new indications with a high success rate in clinical studies, obtaining funding for clinical studies, patent protection, and approval systems. To tackle these obstacles, various approaches have been applied to DR worldwide. In this perspective, we provide representative examples of DR and discuss the ongoing efforts to overcome obstacles to DR in Japan.

OPEN ACCESS

Edited by: Luc Zimmer,

Université Claude Bernard Lyon 1 and Hospices Civils de Lyon, France

Reviewed by:

Afzal Chowdhury,

Perkins Coie, LLP, United States Aris Persidis,

Biovista, United States

*Correspondence:

Yuhei Nishimura

yuhei@doc.medic.mie-u.ac.jp

Specialty section:

This article was submitted to Experimental Pharmacology and Drug

Discovery,

a section of the journal

Frontiers in Pharmacology

Received: 26 July 2017 Accepted: 28 September 2017 Published: 11 October 2017

Citation: Nishimura $Y$, Tagawa $M$, Ito $H$, Tsuruma K and Hara H (2017) Overcoming Obstacles to Drug

Repositioning in Japan.

Front. Pharmacol. 8:729.

doi: 10.3389/fphar.2017.00729
Keywords: industry-sponsored clinical trial, investigator-initiated clinical trial, electronic health record, sharing resources, computational drug repositioning

\section{OBSTACLES TO DR IN JAPAN}

Drug repositioning (DR), also known as "drug repurposing," seeks to develop new indications for existing drugs, to change the formulation, the dosage regimen, and the route of administration, and to create new combinations of drugs directed at multiple therapeutic targets. At present, the conventional de novo drug discovery process requires an average of about 14 years and US\$2.5 billion to approve and launch a drug (Nosengo, 2016). The average time and costs to launch a drug de novo in Japan are 9.2 years and 55 billion yen, respectively (Yagi and Okubo, 2010). For a preclinical and phase-I study, it takes 1.3 and 1.1 billion yen per drug, respectively, in Japan (Yagi and Okubo, 2010). DR usually focuses on drugs that have cleared phase-I safety trials but has yet to show efficacy for the intended indication. Therefore, DR can probably skip the preclinical and phase-I study, thereby reducing the cost throughout drug development. Some estimates suggest that DR requires an average of $\approx 6.5$ years and $\approx$ US $\$ 300$ million to approve and launch a drug (Naylor et al., 2015). However, the cost for a phase-III study remains expensive, and DR is not always a smooth and successful process (Nosengo, 2016). Pharmaceutical companies must have a clear path to economic return to justify the expense of a clinical trial for DR, considering the remaining patent life, new use patents, or data exclusivity (Shineman et al., 2014). This creates an opportunity that foundations and bioventures can help DR (Azvolinsky, 2017). Foundations can directly fund smaller proofof-concept clinical trials for DR, and positive results from such trials may encourage further investment from government and/or industry to fund larger, multicenter trials. Bioventures can provide various pipelines for DR, such as in silico and/or in vitro screening platforms to identify DR candidates with a high success rate. Bioventures can also provide consultation on intellectual property to differentiate the DR candidates from those already marketed. DR can be 
used to obtain patent protection of a class of drugs in an unexpected indication as a method to block competitors from taking advantage of the new finding (e.g., pipeline management protection against competitors) (Mucke and Mucke, 2015). Currently, $\approx 10$ patents related to DR are issued per month (Mucke, 2017). In the United States and Europe, there are many foundations and bioventures supporting DR, including Cure Within Reach (Bloom, 2015), Biovista (Deftereos et al., 2012), Insilico Medicine (Vanhaelen et al., 2017), and H.M. Pharma Consultancy (Mucke and Mucke, 2015). The regulatory frameworks in the US have also been changed to support DR. The US Food and Drug Administration Section 505(b)(2) permits the approval of applications for DR and permits reliance for such approvals on the information from previous studies about safety and/or effectiveness for an approved drug product. Applicants have the possibility to submit a 505(b)(2) application for a previously approved drug product (e.g., to support a new claim). These systems can significantly reduce the cost and effort of clinical trials and stimulate DR in various companies. In contrast, few bioventures support DR in Japan. The Ministry of Health, Labour, and Welfare (MHLW) and the Pharmaceuticals and Medical Devices Agency (PMDA), the regulatory agencies responsible for reviewing applications and approving the marketing authorization of drugs in Japan, do not have regulations equivalent to Section 505(b)(2). Much DR, however, has been successfully performed by both pharmaceutical companies and academia. Between 2001 and 2010, the PMDA gave 463 updated approvals, of which $>60 \%$ were approvals for new indications of existing drugs (Hashitera et al., 2013). In this perspective, we provide representative examples of DR and the ongoing efforts to stimulate DR in Japan.

\section{Zonisamide}

Zonisamide (1,2-benzisoxazole-3-methanesulfonamide) has been developed and marketed in Japan as an anti-epileptic drug by Sumitomo Dainippon Pharma (SDP) since 1989. Murata colleagues used zonisamide to treat epilepsy in a patient with Parkinson's disease (PD) and serendipitously found that not only epilepsy but also symptoms related to PD were improved. They hypothesized that zonisamide could be repositioned for the treatment of PD and subsequently performed investigatorinitiated clinical trials (IIT) in nine patients with PD (Murata et al., 2001). Seven patients showed a clear improvement in PD symptoms. Considering the positive finding, the patent life-cycle management, and the market potential, SDP decided to perform industry-sponsored clinical trials (IST) for the repositioning of zonisamide for PD. Phase IIb/III clinical trial were performed in 320 patients with $\mathrm{PD}$, and patients treated with zonisamide again showed an improvement in PD symptoms. SDP then performed a phase III clinical trial on 133 patients with advanced $\mathrm{PD}$, resulting in positive findings. Although the mechanism of action of zonisamide in both epilepsy and PD has not been fully elucidated, it may differ in each condition because of the 10 -fold difference in approval therapeutic dose. The anti-epilepsy effect of zonisamide has been related to the inhibition of voltage-dependent sodium channels and T-type calcium channels (Holder and Wilfong, 2011). The anti-PD effect of zonisamide, however, may be related to the inhibition of dopamine metabolism via inhibition of monoamine oxidase-B (MAO-B), the stimulation of dopamine release from striatum, and the blockade of T-type calcium channels (Shahed and Jankovic, 2007; Sonsalla et al., 2010; Miwa et al., 2011). Based on these data, zonisamide was approved in Japan as an anti-PD drug under the brand name Trerief ${ }^{\circledR}$ in 2009. The price of Trerief ${ }^{\circledR}$ was determined by MHLW according to the price of selegiline, another anti-PD drug. This method of comparison based on similar efficacy means that the price of Trerief ${ }^{\circledR}$ is significantly higher than that of Excegran $^{\circledR}$, the brand name of zonisamide as an anti-epileptic drug.

\section{Rebamipide}

Rebamipide, a quinolinone derivative, has been developed and marketed in Japan for the treatment of peptic ulcer by Otsuka Pharmaceutical (OP) since 1990. The pharmacodynamics of rebamipide as anti-ulcer drug includes the stimulation of mucin secretion from goblet cells, resulting in the protection of gastric cells from various stimuli by coating the cell surface with a mucinous layer. Mucins are also expressed on the membranes of ocular surface epithelia and are secreted by conjunctival goblet cells, and thus play a role in ocular lubrication and defense. Reduced levels of mucins and changes in their distribution and glycosylation have been reported in patients with dry eye (Vickers and Gupta, 2015). However, therapies for dry eye that functioned by increasing mucin secretion in conjunctiva had not previously been developed. About 35\% of the global population is affected by dry eye and this proportion is increasing, possibly as a result of lifestyle changes such as frequent computer and visual display usage (Vickers and Gupta, 2015). Considering the pharmacological effect of rebamipide on mucin secretion from goblet cells and the demanding need to develop novel therapies for dry eye, OP decided to reposition rebamipide for the treatment of dry eye. They demonstrated that rebamipide increased the production of mucin-like substances in the cornea and conjunctiva of a rabbit model of dry eye. They subsequently performed phase II and III ISTs and successfully demonstrated that a rebamipide ophthalmic suspension (unit dose 2\%) improved both corneal and conjunctival epithelial damage and associated symptoms. Based on these studies, rebamipide was launched in Japan for the treatment of dry eye in 2012 under the brand name Mucosta ${ }^{\circledR}$ ophthalmic suspension UD2\%. The price of this formulation was set at 27 yen per $0.35 \mathrm{ml}$, based on the price of diquafosol sodium (Diquas ${ }^{\circledR}$ ), which was developed by Santen as a de novo treatment for dry eye by increasing mucin secretion via activation of P2Y2 receptors in corneal epithelium (Nakamura et al., 2012). Diquas ${ }^{\circledR}$ has been approved as a drug for dry eye since 2010 in Japan at a price of 641 yen per $5 \mathrm{ml}$, 1.6 times higher than that of Mucosta. A popular Japanese television program has promoted both Mucosta ${ }^{\circledR}$ and Diquas ${ }^{\circledR}$ as effective treatments for dry eye, and Mucosta ${ }^{\circledR}$ has also been used to treat the condition in a well-known Japanese medical comedy show. These advertisements can have a significant 
impact on the public and effectively increase the sales of these drugs.

\section{Carperitide}

Carperitide, a recombinant human atrial natriuretic peptide (ANP), has been used to prevent and/or treat cardiac failure. Nojiri et al. (2012) administered carperitide to patients undergoing lung cancer surgery to prevent post-surgical cardiovascular complications, especially in patients with cardiovascular disease risk factors. However, they serendipitously found that carperitide significantly reduced the metastasis of lung cancer after surgery. They studied the anti-cancer mechanism of carperitide using a mouse model of cancer metastasis and found that it inhibits cancer metastasis through suppression of E-selection in vascular endothelial cells, which decreases the attachment of cancer cells to vascular endothelial cells. They subsequently performed a retrospective study of 467 patients who underwent lung cancer surgery and confirmed that the survival ratio of patients treated with carperitide was significantly higher than that of patients without carperitide treatment. These studies strongly suggest that carperitide can be used to prevent lung cancer metastasis and improve the prognosis of patients with lung cancer (Nojiri et al., 2015). However, the patent for carperitide has expired and many generic ANP therapies are currently in clinical use, with the result that pharmaceutical companies have avoided investing in the repositioning of carperitide as an anti-cancer drug. After prolonged negotiations, Nojiri et al. (2017) have formulated a framework for an IIT that will be performed with the support of Shionogi, a pharmaceutical company based in Japan. The IIT, termed the JANP study, is currently recruiting patients.

\section{Thalidomide}

Thalidomide was initially developed as a sedative and later repositioned to treat erythema nodosum leprosum and multiple myeloma because of its anti-angiogenic and antiinflammatory effects. Kuwabara et al. (2008b) found that polyneuropathy, organomegaly, endocrinopathy, M-protein, and skin changes (POEMS) syndrome could be effectively treated with a combination of intensive chemotherapy and autologous peripheral blood cell-derived stem cell transplantation to suppress the proliferation of monoclonal plasma cells. These treatments, however, cannot be administered to patients with multiple organ failure and/or older age. Kuwabara et al. (2008a) hypothesized that thalidomide could be repositioned for the treatment of POEMS syndrome as it inhibits cytokine production and the proliferation of plasma cells. The authors had previously initiated an IIT in 2006 without pharmaceutical industry support and demonstrated a promising outcome in nine POEMS patients treated with thalidomide, later used as phase II data. Since 2010, Kuwabara et al. have performed a phase II/III IIT, the J-POST study, with the support of Fujimoto Pharmaceutical Corporation, the patent holder of thalidomide for multiple myeloma in Japan (Misawa et al., 2016). The IIT demonstrated that thalidomide reduced serum VEGF concentration and thus represents a novel treatment for patients with POEMS (Misawa et al., 2016). Approval for the use of thalidomide for this indication is pending from the PMDA.

\section{FUTURE DIRECTIONS}

The examples of zonisamide and carperitide presented above demonstrate that serendipitous findings by astute clinicians are an important driver of DR and that the retrospective analysis of clinical records can be used to confirm the validity of these findings. Recent developments in electronic health records (EHRs) have made it possible to identify novel effects of clinical drugs in one EHR database and to validate these findings using other EHR databases (Xu et al., 2015). EHR can also be used to analyze similarity between diseases and between drugs without a prior knowledge of those diseases and drugs (Paik et al., 2015). For example, a novel relationship between disease A and $\mathrm{B}$ and drug $\mathrm{X}$ and $\mathrm{Y}$ can be identified using EHR. If a known relationship exists between disease $\mathrm{A}$ and drug $\mathrm{X}$, one can hypothesize that there may also be a relationship between disease B and drug Y. Therefore, the sharing of EHR data between hospitals and of various clinical trial data in public databases can represent a powerful approach to computational DR. The PMDA is currently developing a public EHR database, Medical Information Database NETwork (MID-NET) (Noguchi, 2015), to be launched in 2018. Data from clinical trials can also be valuable resources for computational DR. Serious adverse events (SAEs) can be identified from randomized clinical trials. If a treatment arm has fewer predefined SAEs than the control arm, it is plausible that the drug used for the treatment arm is reducing the level of SAEs (Su and Sanger, 2017). Sharing data from clinical trials can increase the success rate of DR. The Study Data Tabulation Model (SDTM) from the Clinical Data Interchange Standards Consortium (CDISC) is a standard for creating a "data warehouse" for sharing the data from clinical research across studies. The PMDA has begun to request that the submission of data from clinical trials in accordance with the guidelines of CDISC (Ando, 2016). Tools that can generate SDTM data from clinical trials undertaken already without using CDISC guidelines have also been developed in Japan (Yamamoto et al., 2017). The PMDA and the Japan Agency of Medical Research and Development (AMED) are also developing the Clinical Innovation Network (CIN), a collaboration scheme with national medical research centers and industries, to share the clinical trials data between academia and industry (Hori, 2016). These frameworks may promote DR undertaken first by in silico analyses using big data, such as EHR and clinical-trial data, and then validated in IIT and/or IST.

High throughput screening of chemicals using in vitro and/or in vivo systems has also strongly driven DR (Nishimura and Hara, 2016; Imamura et al., 2017). The sharing of chemicals among researchers with various assay systems can increase the success rate of DR, as demonstrated by the success of projects supported by the National Center for Advancing Translational Sciences in the US (Azvolinsky, 2017). AMED has developed the Drug-Discovery Innovation and Screening Consortium, from 
which over 200,000 chemicals provided by 20 pharmaceutical companies are publicly available. Chemical libraries specialized in DR have also been provided by pharmaceutical companies within open innovation grants. These frameworks may promote DR firstly performed by in vitro/vivo screening, and then evaluated in an IST by the company holding the patent.

A machine-learning approach has also been popular in DR (Napolitano et al., 2013; March-Vila et al., 2017). Various algorithms of machine learning, including random forests (Cao and Moult, 2014), deep neural networks (Aliper et al., 2016), and deep adversarial networks (Kadurin et al., 2017), have been applied to DR combined with various omics databases, such as the Genome Wide Association Study Catalog (MacArthur et al., 2017) and the Library of Integrated Network-based Cellular Signature (LINCS) (Duan et al., 2014). These approaches have also been carried out successfully in Japan. For example, Iwata colleagues undertook an integrative approach using machine learning combined with multiple big data, including LINCS, Connectivity Map (Lamb et al., 2006), a Toxicogenomics ProjectGenomics Assisted Toxicity Evaluation System (Igarashi et al., 2015), ChEMBL (Gaulton et al., 2017), Kyoto Encyclopedia of Genes and Genomes (Kotera et al., 2013), SuperTarget (Gunther et al., 2008), and DrugBank (Law et al., 2014) and found novel drug-protein-disease networks (Iwata et al., 2017). Asako and Uesawa (2017) developed a machine-learning model to predict agonists of the human estrogen receptor based on chemical structures.

The regulatory system for approval can affect the stream of DR significantly. A progressive approval system, in which the drug can be approved following proof of safety (Loike and Miller, 2017), may accelerate DR greatly, as suggested for rare diseases (Dunoyer, 2012) and regenerative medicine (Caplan and West, 2014). However, the risk of eliminating phase-II and -III studies should also be considered carefully (Loike and Miller, 2017). Conditional approval in Europe for

\section{REFERENCES}

Aliper, A., Plis, S., Artemov, A., Ulloa, A., Mamoshina, P., and Zhavoronkov, A. (2016). Deep learning applications for predicting pharmacological properties of drugs and drug repurposing using transcriptomic data. Mol. Pharm. 13, 2524-2530. doi: 10.1021/acs.molpharmaceut.6b00248

Ando, Y. (2016). PMDA Update. Available at: https://www.pmda.go.jp/files/ 000215352.pdf [accessed September 1, 2017].

Asako, Y., and Uesawa, Y. (2017). High-performance prediction of human estrogen receptor agonists based on chemical structures. Molecules 22:E675. doi: 10.3390/ molecules22040675

Azvolinsky, A. (2017). Repurposing Existing Drugs for New Indications. Available at: http://www.the-scientist.com/?articles.view/articleNo/47744/title/ Repurposing-Existing-Drugs-for-New-Indications/ [accessed October 3, 2017].

Bloom, B. E. (2015). Creating new economic incentives for repurposing generic drugs for unsolved diseases using social finance. Assay Drug Dev. Technol. 13, 606-611. doi: 10.1089/adt.2015.29015.beddrrr

Cao, C., and Moult, J. (2014). GWAS and drug targets. BMC Genomics 15(Suppl. 4):S5. doi: 10.1186/1471-2164-15-S4-S5

Caplan, A. I., and West, M. D. (2014). Progressive approval: a proposal for a new regulatory pathway for regenerative medicine. Stem Cells Transl. Med. 3, 560-563. doi: 10.5966/sctm.2013-0180

Deftereos, S. N., Dodou, E., Andronis, C., and Persidis, A. (2012). From depression to neurodegeneration and heart failure: re-examining the potential orphan drugs (Dunoyer, 2012) and an approval system for off-label use of drugs validated in publicly funded research in Japan (Shimazawa and Ikeda, 2012) might serve as templates to consider the progressive approval system for DR. Lifecycle management, which includes patent protection and contributes to maximizing the return of investment for drug discovery, is another important aspect of DR. Analyses of lifecycle management in the Japanese market are undertaken actively (Hashitera et al., 2013; Yamanaka and Kano, 2016).

\section{AUTHOR CONTRIBUTIONS}

YN conceived and wrote the paper. MT, HI, KT, and $\mathrm{HH}$ wrote the paper.

\section{FUNDING}

This work was supported in part by the Japan Society for the Promotion of Science KAKENHI (16K08547), the Long-range Research Initiative of the Japan Chemical Industrial Association (13-PT01-01), and Okasan-Kato Foundation.

\section{ACKNOWLEDGMENTS}

The authors would like to thank Dr. Kazutaka Ikeda (Tokyo Metropolitan Institute of Medical Science) for his valuable comments and suggestions. We sincerely apologize to all those researchers and stakeholders whose important works are not cited because of space considerations. We also thank Clare Cox, Ph.D., from Edanz Group (www.edanzediting.com/ac) for editing a draft of this manuscript.

of MAO inhibitors. Expert Rev. Clin. Pharmacol. 5, 413-425. doi: 10.1586/ecp. 12.29

Duan, Q., Flynn, C., Niepel, M., Hafner, M., Muhlich, J. L., Fernandez, N. F., et al. (2014). LINCS Canvas Browser: interactive web app to query, browse and interrogate LINCS L1000 gene expression signatures. Nucleic Acids Res. 42, W449-W460. doi: 10.1093/nar/ gku476

Dunoyer, M. (2012). A Modern Progressive Approval System for Rare Diseases. Available at: www.raps.org/WorkArea/DownloadAsset.aspx?id=4398 [accessed September 1, 2017].

Gaulton, A., Hersey, A., Nowotka, M., Bento, A. P., Chambers, J., Mendez, D., et al. (2017). The ChEMBL database in 2017. Nucleic Acids Res. 45, D945-D954. doi: 10.1093/nar/gkw1074

Gunther, S., Kuhn, M., Dunkel, M., Campillos, M., Senger, C., Petsalaki, E., et al. (2008). SuperTarget and Matador: resources for exploring drug-target relationships. Nucleic Acids Res. 36, D919-D922.

Hashitera, Y., Saotome, C., and Yamamoto, H. (2013). Analysis of 10 years drug lifecycle management (LCM) activities in the Japanese market. Drug Discov. Today 18, 1109-1116. doi: 10.1016/j.drudis.2013. 07.004

Holder, J. L. Jr., and Wilfong, A. A. (2011). Zonisamide in the treatment of epilepsy. Expert Opin. Pharmacother. 12, 2573-2581. doi: 10.1517/14656566.2011. 622268 
Hori, A. (2016). PMDA Perspective:Utilization of the Disease Registry Data for Drug Development. Available at: https://www.pmda.go.jp/files/000215446.pdf [assessed September 3, 2017].

Igarashi, Y., Nakatsu, N., Yamashita, T., Ono, A., Ohno, Y., Urushidani, T., et al. (2015). Open TG-GATEs: a large-scale toxicogenomics database. Nucleic Acids Res. 43, D921-D927. doi: 10.1093/nar/ gku955

Imamura, K., Izumi, Y., Watanabe, A., Tsukita, K., Woltjen, K., Yamamoto, T., et al. (2017). The Src/c-Abl pathway is a potential therapeutic target in amyotrophic lateral sclerosis. Sci. Transl. Med. 9:eaaf3962. doi: 10.1126/scitranslmed. aaf3962

Iwata, M., Sawada, R., Iwata, H., Kotera, M., and Yamanishi, Y. (2017). Elucidating the modes of action for bioactive compounds in a cell-specific manner by large-scale chemically-induced transcriptomics. Sci. Rep. 7:40164. doi: 10.1038/ srep40164

Kadurin, A., Nikolenko, S., Khrabrov, K., Aliper, A., and Zhavoronkov, A. (2017). druGAN: an advanced generative adversarial autoencoder model for de novo generation of new molecules with desired molecular properties in silico. Mol. Pharm. 14, 3098-3104. doi: 10.1021/acs.molpharmaceut. $7 \mathrm{~b} 00346$

Kotera, M., Tabei, Y., Yamanishi, Y., Moriya, Y., Tokimatsu, T., Kanehisa, M., et al. (2013). KCF-S: KEGG chemical function and substructure for improved interpretability and prediction in chemical bioinformatics. BMC Syst. Biol. 7(Suppl. 6):S2. doi: 10.1186/1752-0509-7-S6-S2

Kuwabara, S., Misawa, S., Kanai, K., Sawai, S., Hattori, T., Nishimura, M., et al. (2008a). Thalidomide reduces serum VEGF levels and improves peripheral neuropathy in POEMS syndrome. J. Neurol. Neurosurg. Psychiatry 79, 1255-1257. doi: 10.1136/jnnp.2008.150177

Kuwabara, S., Misawa, S., Kanai, K., Suzuki, Y., Kikkawa, Y., Sawai, S., et al. (2008b). Neurologic improvement after peripheral blood stem cell transplantation in POEMS syndrome. Neurology 71, 1691-1695. doi: 10.1212/ 01.wnl.0000323811.42080.a4

Lamb, J., Crawford, E. D., Peck, D., Modell, J. W., Blat, I. C., Wrobel, M. J., et al. (2006). The connectivity map: using gene-expression signatures to connect small molecules, genes, and disease. Science 313, 1929-1935. doi: 10.1126/ science.1132939

Law, V., Knox, C., Djoumbou, Y., Jewison, T., Guo, A. C., Liu, Y., et al. (2014). DrugBank 4.0: shedding new light on drug metabolism. Nucleic Acids Res. 42, D1091-D1097. doi: 10.1093/nar/gkt1068

Loike, J., and Miller, J. (2017). Improving FDA Evaluations Without Jeopardizing Safety and Efficacy. Available at: http://www.the-scientist.com/?articles.view/ articleNo/48280/title/Opinion-Improving-FDA-Evaluations-WithoutJeopardizing-Safety-and-Efficacy/ [accessed October 3, 2017].

MacArthur, J., Bowler, E., Cerezo, M., Gil, L., Hall, P., Hastings, E., et al. (2017). The new NHGRI-EBI catalog of published genome-wide association studies (GWAS Catalog). Nucleic Acids Res. 45, D896-D901. doi: 10.1093/nar/ gkw1133

March-Vila, E., Pinzi, L., Sturm, N., Tinivella, A., Engkvist, O., Chen, H., et al. (2017). On the integration of in silico drug design methods for drug repurposing. Front. Pharmacol. 8:298. doi: 10.3389/fphar.2017. 00298

Misawa, S., Sato, Y., Katayama, K., Nagashima, K., Aoyagi, R., Sekiguchi, Y., et al. (2016). Safety and efficacy of thalidomide in patients with POEMS syndrome: a multicentre, randomised, double-blind, placebocontrolled trial. Lancet Neurol. 15, 1129-1137. doi: 10.1016/S1474-4422(16) 30157-0

Miwa, H., Koh, J., Kajimoto, Y., and Kondo, T. (2011). Effects of T-type calcium channel blockers on a parkinsonian tremor model in rats. Pharmacol. Biochem. Behav. 97, 656-659. doi: 10.1016/j.pbb.2010.11.014

Mucke, H. A. (2017). Patent highlights December 2016-January 2017. Pharm. Pat. Anal. 6, 97-104. doi: 10.4155/ppa-2017-0007

Mucke, H. A., and Mucke, E. (2015). Sources and targets for drug repurposing: landscaping transitions in therapeutic space. ASSAY Drug Dev. Technol. 13, 319-324. doi: 10.1089/adt.2015.29009.hmedrrr

Murata, M., Horiuchi, E., and Kanazawa, I. (2001). Zonisamide has beneficial effects on Parkinson's disease patients. Neurosci. Res. 41, 397-399. doi: 10.1016/ S0168-0102(01)00298-X
Nakamura, M., Imanaka, T., and Sakamoto, A. (2012). Diquafosol ophthalmic solution for dry eye treatment. Adv. Ther. 29, 579-589. doi: 10.1007/s12325012-0033-9

Napolitano, F., Zhao, Y., Moreira, V. M., Tagliaferri, R., Kere, J., D’amato, M., et al. (2013). Drug repositioning: a machine-learning approach through data integration. J. Cheminform. 5:30. doi: 10.1186/1758-294 6-5-30

Naylor, S., Kauppi, D., and Schonfeld, J. (2015). Therapeutic Drug Repurposing, Repositioning and Rescue Part III Market Exclusivity Using Intellectual Property and Regulatory Pathways. Available at: http://www.ddw-online.com/ drug-discovery/p303678-therapeutic-drug-repurposingrepositioning-andrescue-part-iiimarket-exclusivity-using-intellectual-property-and-regulatorypathways.html [accessed September 3, 2017].

Nishimura, Y., and Hara, H. (2016). Integrated approaches to drug discovery for oxidative stress-related retinal diseases. Oxid. Med. Cell Longev. 2016, 1-9. doi: $10.1155 / 2016 / 2370252$

Noguchi, A. (2015). Challenges for Post-marketing Drug Safety Measures Using Electronic Healthcare Database in Japan. Available at: https://www.pmda.go.jp/ files/000215551.pdf [assessed September 3, 2017].

Nojiri, T., Hosoda, H., Tokudome, T., Miura, K., Ishikane, S., Otani, K., et al. (2015). Atrial natriuretic peptide prevents cancer metastasis through vascular endothelial cells. Proc. Natl. Acad. Sci. U.S.A. 112, 4086-4091. doi: 10.1073/ pnas. 1417273112

Nojiri, T., Inoue, M., Yamamoto, K., Maeda, H., Takeuchi, Y., Funakoshi, Y., et al. (2012). Effects of low-dose human atrial natriuretic peptide for preventing post-operative cardiopulmonary complications in elderly patients undergoing pulmonary resection for lung cancer. Eur. J. Cardiothorac. Surg. 41, 1330-1334. doi: $10.1093 /$ ejcts/ezr202

Nojiri, T., Yamamoto, H., Hamasaki, T., Onda, K., Ohshima, K., Shintani, Y., et al. (2017). A multicenter randomized controlled trial of surgery alone or surgery with atrial natriuretic peptide in lung cancer surgery: study protocol for a randomized controlled trial. Trials 18, 183. doi: 10.1186/s13063-0171928-1

Nosengo, N. (2016). Can you teach old drugs new tricks? Nature 534, 314-316. doi: $10.1038 / 534314 a$

Paik, H., Chung, A. Y., Park, H. C., Park, R. W., Suk, K., Kim, J. et al. (2015). Repurpose terbutaline sulfate for amyotrophic lateral sclerosis using electronic medical records. Sci. Rep. 5:8580. doi: 10.1038/ srep 08580

Shahed, J., and Jankovic, J. (2007). Exploring the relationship between essential tremor and Parkinson's disease. Parkinsonism Relat. Disord. 13, 67-76. doi: 10.1016/j.parkreldis.2006.05.033

Shimazawa, R., and Ikeda, M. (2012). Japanese regulatory system for approval of off-label drug use: evaluation of safety and effectiveness in literaturebased applications. Clin. Ther. 34, 2104-2116. doi: 10.1016/j.clinthera.2012. 09.004

Shineman, D. W., Alam, J., Anderson, M., Black, S. E., Carman, A. J., Cummings, J. L., et al. (2014). Overcoming obstacles to repurposing for neurodegenerative disease. Ann. Clin. Transl. Neurol. 1, 512-518. doi: 10.1002/ $\operatorname{acn} 3.76$

Sonsalla, P. K., Wong, L. Y., Winnik, B., and Buckley, B. (2010). The antiepileptic drug zonisamide inhibits MAO-B and attenuates MPTP toxicity in mice: clinical relevance. Exp. Neurol. 221, 329-334. doi: 10.1016/j.expneurol.2009. 11.018

Su, E. W., and Sanger, T. M. (2017). Systematic drug repositioning through mining adverse event data in ClinicalTrials.gov. PeerJ 5:e3154. doi: 10.7717/peerj. 3154

Vanhaelen, Q., Mamoshina, P., Aliper, A. M., Artemov, A., Lezhnina, K., Ozerov, I., et al. (2017). Design of efficient computational workflows for in silico drug repurposing. Drug Discov. Today 22, 210-222. doi: 10.1016/j.drudis.2016.09. 019

Vickers, L. A., and Gupta, P. K. (2015). The future of dry eye treatment: a glance into the therapeutic pipeline. Ophthalmol. Ther. 4, 69-78. doi: 10.1007/s40123015-0038-y

Xu, H., Aldrich, M. C., Chen, Q., Liu, H., Peterson, N. B., Dai, Q., et al. (2015). Validating drug repurposing signals using electronic health records: a case study of metformin associated with reduced cancer mortality. 
J. Am. Med. Inform. Assoc. 22, 179-191. doi: 10.1136/amiajnl-2014002649

Yagi, T., and Okubo, M. (2010). JPMA News Letter. Available at: http://www.jpma. or.jp/about/issue/gratis/newsletter/archive_until2014/pdf/2010_136_12.pdf [accessed September 3, 2017].

Yamamoto, K., Ota, K., Akiya, I., and Shintani, A. (2017). A pragmatic method for transforming clinical research data from the research electronic data capture "REDCap" to Clinical Data Interchange Standards Consortium (CDISC) Study Data Tabulation Model (SDTM): Development and evaluation of REDCap2SDTM. J. Biomed. Inform. 70, 65-76. doi: 10.1016/j.jbi.2017.05.003

Yamanaka, T., and Kano, S. (2016). Patent term extension systems differentiate Japanese and US drug lifecycle management. Drug Discov. Today 21, 111-117. doi: 10.1016/j.drudis.2015.09.005
Conflict of Interest Statement: MT and HI have been employed by Sumitomo Dainippon Pharma and Otsuka Pharmaceutical, respectively.

The other authors declare that the research was conducted in the absence of any commercial or financial relationships that could be construed as a potential conflict of interest.

Copyright (c) 2017 Nishimura, Tagawa, Ito, Tsuruma and Hara. This is an open-access article distributed under the terms of the Creative Commons Attribution License (CC BY). The use, distribution or reproduction in other forums is permitted, provided the original author(s) or licensor are credited and that the original publication in this journal is cited, in accordance with accepted academic practice. No use, distribution or reproduction is permitted which does not comply with these terms. 